\title{
Does the age at disease onset cause a delay in diagnosis and affect disease severity in the children with familial Mediterranean fever?
}

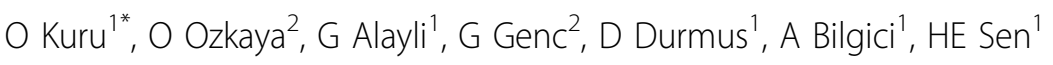 \\ From 8th International Congress of Familial Mediterranean Fever and Systemic Autoinflammatory Diseases \\ Dresden, Germany. 30 September - 3 October 2015
}

\begin{abstract}
Introduction
Familial Mediterranean fever (FMF) is a systemic autoinflammatory disease characterized by recurrent attacks of fever and sterile peritonitis, pleuritis, arthritis or erysipelas-like erythema. Symptoms related with FMF can begin at very early ages of life. However, since most symptoms related to FMF are often well-known only when children become more verbal, and fever in children in the first years of life are common, attacks of fever alone may cause or contribute to diagnosis delay.
\end{abstract}

\section{Objectives}

In the present study we aimed to investigate whether the age at onset of the symptoms cause a diagnosis delay in the children with FMF.

\section{Materials and methods}

Ninety FMF subjects (M/F: 43/47) who fulfilled the Livneh criteria for the diagnosis of FMF were enrolled into the study. Subjects were between 8-18 years age and having the symptoms of FMF for at least one year. Demographic data including age, age at onset of the symptoms, duration of symptoms and family history were recorded. The subjects were questioned about periodic fever, abdominal and chest pain, arthritis, and erysipelas-like erythema. The severity score of the disease was calculated based on the Tel-Hashomer severity score. 6 Minute Walk Test (6MWT) was used to evaluate functional capacity.

\section{Results}

The mean age of the FMF subjects was $12.21 \pm 2.75$ years. Abdominal pain attacks were the most common

${ }^{1}$ Ondokuz Mayis University, Medical Faculty, Physical Medicine and Rehabilitation, Samsun, Turkey

Full list of author information is available at the end of the article symptom, occurring in 77 (85.6\%) of our patients; periodic fever was in $74(82.2 \%)$, chest pain in $36(40 \%)$, arthritis in 41 (45.6\%), and erysipelas-like erythema in 12 (13.3\%). A positive family history for FMF was detected in $51(56.6 \%)$ of the patients. Onset age and diagnosis delay duration did not significantly differ between the groups regarding family history $(\mathrm{p}>0.05)$. Twenty-one children (23.3\%) had the first FMF symptoms at the age of $<5$ years. While the mean onset age was $6.91 \pm 3.44$ years, delay in diagnosis was $2.12 \pm 2.70$ years. In the children with FMF, while there was a negative correlation between the age at onset of the symptoms and duration of delay and disease severity score, there was no correlation between 6MWT.

\section{Conclusion}

We determined that FMF beginning at early ages causes a delay in diagnosis and also a worse disease severity score. Physicians practice in the regions that FMF is prevalent should be aware of different features of the disease in childhood in order to reduce a possible delay in diagnosis and prevent the development of complications.

\section{Authors' details}

${ }^{1}$ Ondokuz Mayis University, Medical Faculty, Physical Medicine and Rehabilitation, Samsun, Turkey. ${ }^{2}$ Ondokuz Mayis University, Medical Faculty, Pediatric Nephrology, Samsun, Turkey.

Published: 28 September 2015

doi:10.1186/1546-0096-13-S1-P75

Cite this article as: Kuru et al:: Does the age at disease onset cause a delay in diagnosis and affect disease severity in the children with familial Mediterranean fever? Pediatric Rheumatology 2015 13(Suppl 1): P75. 